\title{
Developmental Changes in Calcium Conductances Contribute to the Physiological Maturation of Cerebellar Purkinje Neurons in Culture
}

\author{
D. L. Gruol, C. R. Deal, and A. J. Yool \\ Department of Neuropharmacology, The Scripps Research Institute, La Jolla, California 92037
}

The relationship between calcium conductances and developmental changes in the active and passive membrane properties of cerebellar Purkinje neurons from rats was studied in a culture model system by using current-clamp and voltage-clamp techniques. These cultures, at 6-21 d of age, represented the main period of morphological and physiological development of the Purkinje neuron. In the currentclamp studies, input resistance decreased and the currentvoltage curve became more S-shaped as the neurons matured in culture. Spike-generating properties also changed. Immature Purkinje neurons without dendritic structure produced repetitive, fast TTX-sensitive simple spikes when stimulated electrically. The simple spike frequency increased with maturation. In older neurons ( $\geq 12 \mathrm{~d}$ in vitro) with well-developed dendritic structure, a burst event, the complex spike, preceded the repetitive simple spike firing. Magnesium (10 $\mathrm{mm}$ ) and cadmium (50-100 $\mu \mathrm{M}$ ), calcium channel blockers, antagonized the repetitive simple spike firing in both young and old neurons. The complex spike of the older neurons was also antagonized by magnesium (10 $\mathrm{mM}$ ) but was resistant to cadmium (50-100 $\mu \mathrm{M})$, suggesting that a pharmacologically distinct calcium conductance mediated this spike event. Whole-cell voltage-clamp recordings showed that the older Purkinje neurons expressed two calcium currents, a low-threshold rapidly inactivating calcium current resistant to cadmium (50-100 $\mu \mathrm{M})$ and a high-threshold slowly inactivating calcium current antagonized by cadmium (50-100 $\mu \mathrm{M}$ ). In young Purkinje neurons without dendritic structure (6-9 d in vitro), only the high-threshold calcium current was evident. The amplitude of this current increased $\sim 50 \%$ during development. These results indicate that the developmental expression of calcium conductances plays a prominent role in the physiological maturation of the cultured Purkinje neurons, which closely simulate the physiologic cells they model. The high-threshold calcium conductance is expressed early in development and contributes to repetitive simple spike firing of both the young and old neurons. The low-threshold calcium conductance appears later in development, coincident with dendritic expression, and plays a major role in the generation of the complex spike.

\footnotetext{
Received Dec. 20, 1991; accepted Mar. 2, 1992.

This work was supported by NIH Grant NS21777 to D.L.G. and NIAAA Training Grant AA07456.

Correspondence should be addressed to Dr. Donna Gruol, Department of Neuropharmacology, The Scripps Research Institute, 10666 North Torrey Pines Road, La Jolla, CA 92037.

- Present address: Beckman Center, Department of Molecular and Cellular Physiology, Stanford University Medical Center, Stanford, CA 94305.

Copyright (C) 1992 Society for Neuroscience $0270-6474 / 92 / 122838-11 \$ 05.00 / 0$
}

During the first 3 weeks of postnatal development in the rat, cerebellar Purkinje neurons grow from simple immature cells to large neurons with extensive dendritic arborization (Altman, 1972). Equally dramatic changes in functional properties occur during this period. Initially inexcitable, the Purkinje neurons eventually express spontaneous high-frequency firing patterns and two types of spikes, simple and complex (Woodward et al., 1969a,b; Crepel, 1972). The spontaneous firing begins during the first postnatal week, as a slow irregular pattern of simple and doublet spikes. Increases in rate and regularity with age yield the characteristic high-frequency firing patterns of the mature neurons. Burst discharges, thought to reflect spontaneous complex spikes initiated by synaptic input from the climbing fibers, appear at 7-9 d postnatal and contribute to the adult pattern at a rate of $1-2 \mathrm{~Hz}$.

These changes in spontaneous activity presumably reflect alterations in the active and passive membrane properties of the Purkinje neuron and in synaptic input. However, the association between functional changes in membrane properties or synaptic input and the developmental process has not been examined. Expression of dendritic structure, in particular, should have a pronounced effect on the electrophysiological properties of the developing Purkinje neuron. The dendrites are the main site of synaptic input and possess many voltage-sensitive conductances including calcium conductances, which are known to play a prominent role in the generation of spontaneous firing patterns and the complex spike of mature Purkinje neurons (Llinas and Sugimori, 1980b; Crepel et al., 1981; Ross and Werman, 1987; Tank et al., 1988; Bossu et al., 1989, 1990; Chan et al., 1989; Hockberger et al., 1989a,b; Hounsgaard and Midtgaard, 1990; Gruol et al., 1991).

The small size of immature neurons, the difficulty in identifying cell types, and the delicate nature of CNS tissue can hamper electrophysiological studies of CNS neuronal development in vivo or in acutely isolated tissues. Culture preparations provide an alternative approach for such studies. Immature Purkinje neurons when transplanted into an appropriate culture environment continue their maturation in vitro where they can be studied electrophysiologically (Seil, 1979; Marshall et al., 1980; Moonen et al., 1982; Weber and Schachner, 1984; Calvet et al., 1985; Hirano and Ohmori, 1986; Gruol and Franklin, 1987; Kapoor et al., 1988; Gahwiler and Llano, 1989; Hockberger et al., 1989a). In our modified organotypic culture preparation, developmental changes in morphology and firing properties generally parallel that observed in vivo (Gruol and Franklin, 1987). For example, the immature Purkinje neurons in culture initially lack spontaneous activity, but later express this activity, eventually exhibiting high-frequency patterns of both simple and complex spikes that, although somewhat lower in rate, resem- 
bled the patterns found in vivo. The early firing patterns, which appear around 1 week in culture, consist of irregular patterns of simple and doublet spikes, similar to that observed in vivo. Firing rate and regularity increase with culture age. By 2 weeks in culture, spontaneous burst events that resembled complex spikes of the in vivo neurons are evident. The similarities of these developmental changes to those observed in vivo suggest that expression of intrinsic membrane properties follows a similar time course in the two systems. Thus, we have used this culture system as a model in which to study the active and passive membrane properties of the Purkinje neuron during maturation. The contribution of calcium conductances to these properties was emphasized because of their prominent role in the generation of spontaneous activity and the complex spikes of the mature neurons. Our results indicate that developmental changes in calcium conductances strongly influence the electrophysiological propcrtics of Purkinje neurons as they pass from immaturity to maturity.

\section{Materials and Methods}

Culture methods

Cultures were prepared as described previously (Gruol, 1983). Briefly, the cortical region of $20 \mathrm{~d}$ embryonic rat cerebella were isolated, minced, triturated, and plated on poly-D-lysine-coated tissue culture dishes containing minimal essential medium (MEM) with $10 \%$ fetal calf serum and $10 \%$ horse serum. The cultures were incubated at $37^{\circ} \mathrm{C}$ in a $5 \% \mathrm{CO}_{2}$ humidified atmosphere. Media was changed twice weekly with MEM containing $10 \%$ horse serum. The cultures were briefly treated with 5 -fluorodeoxyuridine $(20 \mu \mathrm{g} / \mathrm{ml})$ to retard the growth of non-neuronal cells.

\section{Electrophysiological methods}

The Purkinje neurons were identified by morphological criteria previously confirmed by immunohistochemical labeling (Gruol and Crimi, 1988). Current-clamp and voltage-clamp recordings were made in the somatic region using patch electrodes prepared from hematocrit capillary tubes (Fisher Scientific) coated with Sylgard and heat-polished before use. Experiments were carried out at room temperature $\left(\sim 20^{\circ} \mathrm{C}\right)$.

Current-clamp studies. Whole-cell current-clamp recordings were made with the Axopatch-1B (Axon Instruments) amplifier. The recording electrodes (5-6 $\mathrm{M} \Omega$ resistances) contained a high- $\mathrm{K}^{+}$saline of the following composition (in $\mathrm{mM}$ ): $\mathrm{K}^{+}$-gluconate, $154 ; \mathrm{NaCl}, 6 ; \mathrm{MgCl}_{2}, 2$; $\mathrm{CaCl}_{2}, 0.85$; EGTA, 1 ; glucose, 10 ; HEPES-NaOH, 10 (pH 7.3). In most experiments, the recording chamber contained physiological saline of the following composition (in $\mathrm{mM}$ ): $\mathrm{NaCl}, 140 ; \mathrm{KCl}, 3.5 ; \mathrm{KH}_{2} \mathrm{PO}_{4}, 0.4$; $\mathrm{Na}_{2} \mathrm{HPO}_{4}, 1.25 ; \mathrm{CaCl}_{2}, 2.2 ; \mathrm{MgSO}_{4}$ or $\mathrm{MgCl}_{2}, 2$; glucose, $10 ;$ HEPES$\mathrm{NaOH}, 10$ (pH 7.3). Salts containing phosphates or sulfates were eliminated in some studies. Recordings were monitored on a polygraph and oscilloscope. Selected data were recorded on FM tape (Racal Store 4DS recorder) for later playback onto a polygraph recorder at reduced tape speed for better resolution of fast events. The polygraph recordings were used for manual analysis of data and to prepare the figures.

Voltage-clamp studies. Whole-cell voltage-clamp recordings were made with the Axoclamp-2 amplifier (Axon Instruments) using the single electrode switching mode. Switching frequencies ranged from 10 to 15 $\mathrm{kHz}$. Gains were usually around 3. Current recordings were filtered at $0.3-1 \mathrm{kHz}$. Clamp parameters were optimized while viewing the unfiltered headstage output and the current and voltage outputs during a 20 msec $20 \mathrm{mV}$ square-wave voltage command. Clamp parameters were set at the start of each cell recording and adjusted thereafter to maintain optimal conditions.

Calcium currents were studied in isolation under conditions where calcium was the main permeant cation and pharmacological blockers minimized the contribution of other conductances to the measured current. Usually the patch electrodes (3-5 M resistances) contained saline of the following composition (in mM): $\mathrm{CsCl}$ or $\mathrm{CsMeSO}_{3}, 154$; $\mathrm{NaCl}, 6 ; \mathrm{MgCl}_{2}, 2 ; \mathrm{CaCl}_{2}, 0.85$; EGTA, 1; glucose, 10; HEPES-NaOH, 10 (pH 7.3). The saline in the recording chamber contained (in $\mathrm{mM}$ ) $N$-methyl-D-glucamine, 111 (titrated to $\mathrm{pH} 7.3$ with $\mathrm{HCl}$, gluconic acid, or methanesulfonic acid); $\mathrm{KH}_{2} \mathrm{PO}_{4}, 0.4 ; \mathrm{Na}_{2} \mathrm{HPO}_{4}, 1.25 ; \mathrm{CaCl}_{2}, 4.2$; $\mathrm{MgSO}_{4}$ or $\mathrm{MgCl}_{2}, 2$; glucose, 10 ; tetraethylammonium chloride or acetate, 20; 4-aminopyridine, 2; $\mathrm{CsCl}$, 2; glucose, 10; and HEPES- $\mathrm{NaOH}$, 10 (pH 7.3). In some experiments, salts containing phosphates or sulfates were eliminated.

pCLAMP programs and the Labmaster interface (Axon instruments) were used for data acquisition and analysis. Data were digitized at 2 $\mathrm{kHz}$. Currents were evoked from holding potentials of approximately $-90 \mathrm{mV},-62 \mathrm{mV}$, and $-40 \mathrm{mV}$ using a series of test commands 250 $\mathrm{msec}$ in duration applied at a frequency of $0.2 \mathrm{~Hz}$. Obvious escape from space clamp occurred in some cells at some test potentials, as evident by long tail currents and irregularities in the voltage trace. Data from these potentials have been eliminated except where noted. The high input resistance of the neurons ( $>1 \mathrm{G} \Omega$; estimated from hyperpolarizing voltage commands) under the conditions used helped reduce spaceclamp problems. Leak subtraction was not used because of the small size of the leak currents (e.g., see Fig. 10). Initial tests showed that only minor differences in current amplitudes were evident when data obtained using leak subtraction were compared to data obtained without this manipulation in the same cell. Capacitative artifacts were removed manually from current records shown.

In addition to the inward calcium currents, a residue outward current remained under the conditions used, presumably resulting from cesium flux through potassium channels resistant to the channel blockers used. The contribution of this current to the recorded current affected the reversal potential for the calcium currents, which was more hyperpolarized than would be expected based on the ionic conditions used. When the calcium currents were blocked using calcium channel blockers, the outward current activated at potentials around $0 \mathrm{mV}$ and increased in amplitude as a function of membrane depolarization. Thus, calcium currents measured at membrane potentials ranging from $-80 \mathrm{mV}$ to 0 $\mathrm{mV}$ were only minimally affected by the residue outward current. Interpretations of results were limited to that voltage range.

Currents often increased in magnitude during the first few minutes of a recording period. This initial increase could be due to intracellular dialysis of the cesium solution in the recording electrode, which would block potassium conductances, or due to washout of intracellular components. To control for these changes, all data were collected in the same manner using the same sequence of holding potentials, $\sim-62 \mathrm{mV}$, $-90 \mathrm{mV}$, and $-40 \mathrm{mV}$. Data collection was initiated $\sim 1 \mathrm{~min}$ after obtaining a recording. Studies with antagonists were pursued after the current-voltage data were obtained.

Drug application. Ion channel blockers were added directly to the recording chamber or applied by micropressure from drug pipettes $(\sim 2$ $\mu \mathrm{m}$ tip diameter). Results were similar with both methods. Ion channel blockers used included cadmium chloride, nickel chloride, magnesium chloride, amiloride, cesium chloride, and tetrodotoxin (TTX).

Statistical analyses. All compiled data are expressed as mean \pm SEM; $n$ is the number of neurons studied. Statistical significance was determined by one-way analysis of variance (ANOVA) followed by the Fisher's protected least-significant difference (PLSD) test for multiple comparisons. Results reported were based on current-clamp recordings from 55 Purkinje neurons and voltage-clamp recordings from 50 Purkinje ncurons.

\section{Results}

\section{Current-clamp studies}

Developmental changes in the active and passive membrane properties of cultured Purkinje neurons were characterized by features of the current-evoked voltage responses at five culture ages encompassing the main period of morphological development: $6-7,9-10,12-13,14-16$, and $>20 \mathrm{~d}$ in vitro (DIV). At 6-7 DIV, the Purkinje neurons were morphologically immature, consisting of a large soma, an axon, and several fine perisomatic processes (Fig. 1). By 9-10 DIV, most of the perisomatic processes had disappeared and dendritic structure was evident. By 12-13 DIV, one or more branching dendrites were present. By 16 DIV, well-developed dendritic structure was common. Typical recordings from Purkinje neurons at these culture ages are shown in Figure 2. Differences in the number and types of spikes evoked and the amplitude of the voltage 

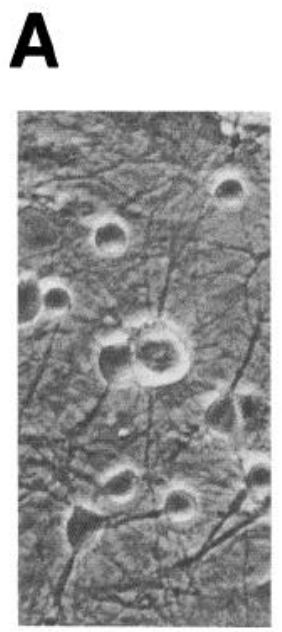
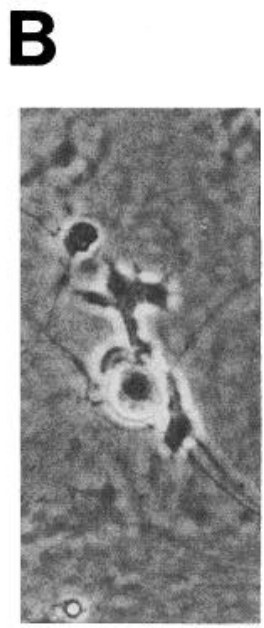
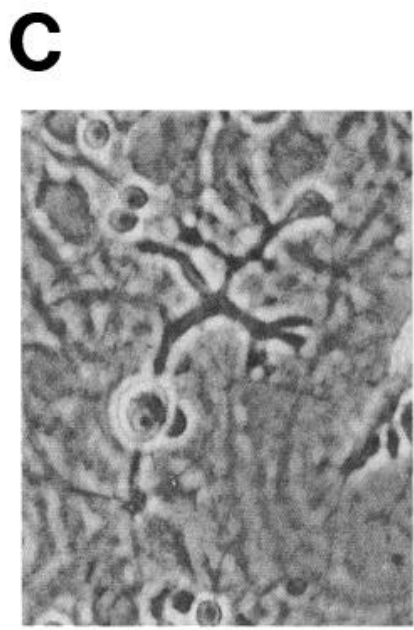

D

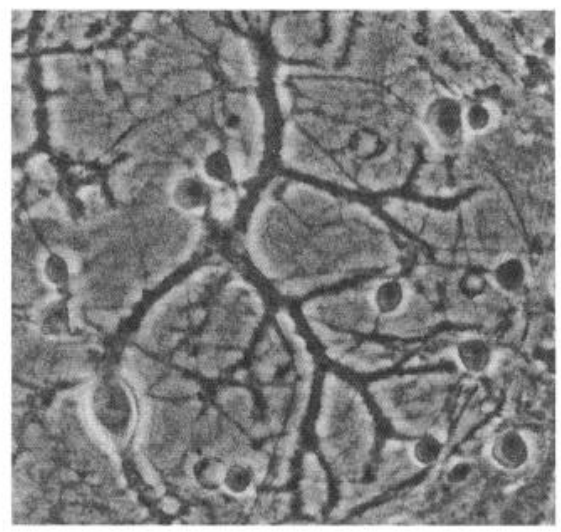

Figure 1. Morphological development of cerebellar Purkinje neurons in culture. These phase-contrast micrographs show the typical morphology of Purkinje neurons at 6-7 DIV $(A)$, 9-10 DIV $(B)$, 12-16 DIV $(C)$, and >16 DIV $(D)$. At the youngest culture age, the Purkinje neurons have no dendritic structure. Dendritic structure develops with age in culture, resulting in a large increase in cell size. Scale bar, $20 \mu \mathrm{m}$.

responses indicated that developmental changes in the active and passive membrane properties occurred during this period.

\section{Response to depolarizing current pulses}

At all culture ages, depolarizing current pulses of sufficient amplitude evoked repetitive simple spike firing, which increased in frequency to a maximum with current intensity (Figs. 2, 3A).
The maximum firing rate attained increased between 9-10 and 12-13 DIV (Fig. 3B). In some young neurons, but more commonly in the older neurons, a burst event preceded the repetitive simple spike firing (Fig. 2). The burst event consisted of a slow depolarization with two or three superimposed fast spikes. In older neurons, the burst event resembled the complex spike of mature Purkinje neurons recorded in vivo or in the slice prep-
7 DIV
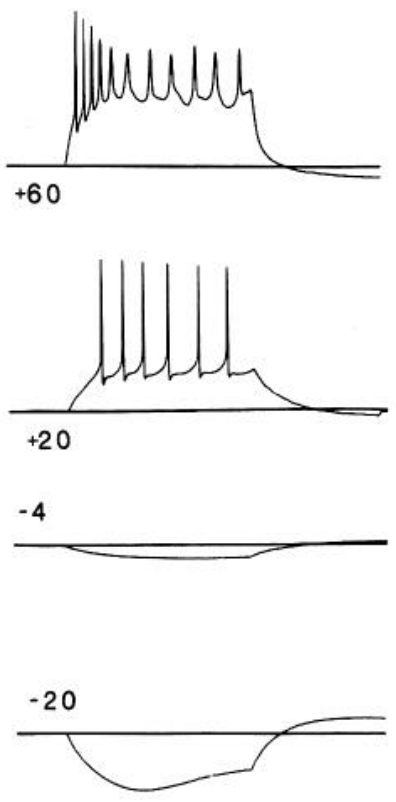

10 DIV

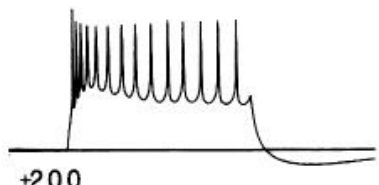

$+200$
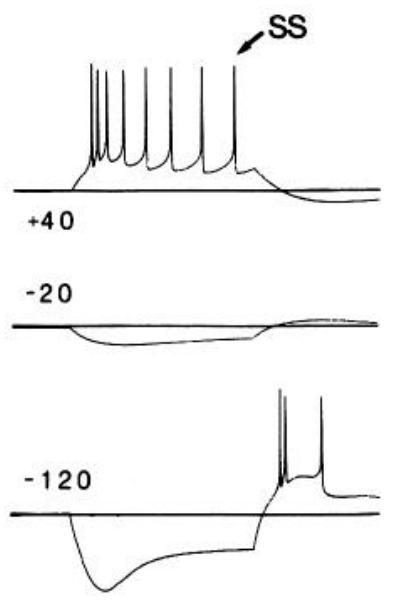

12 DIV

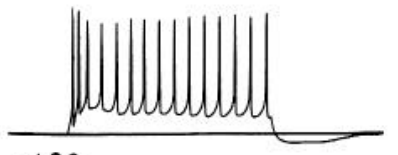

$+160$

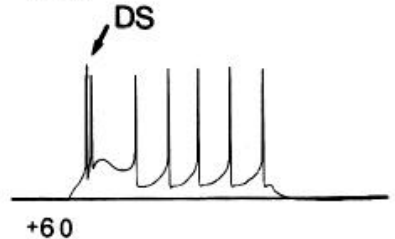

$+60$

$-20$

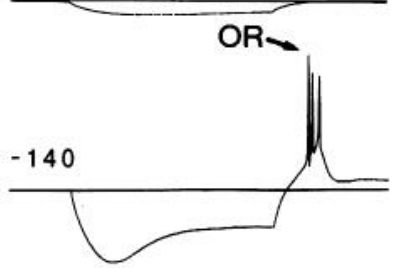

21 DIV
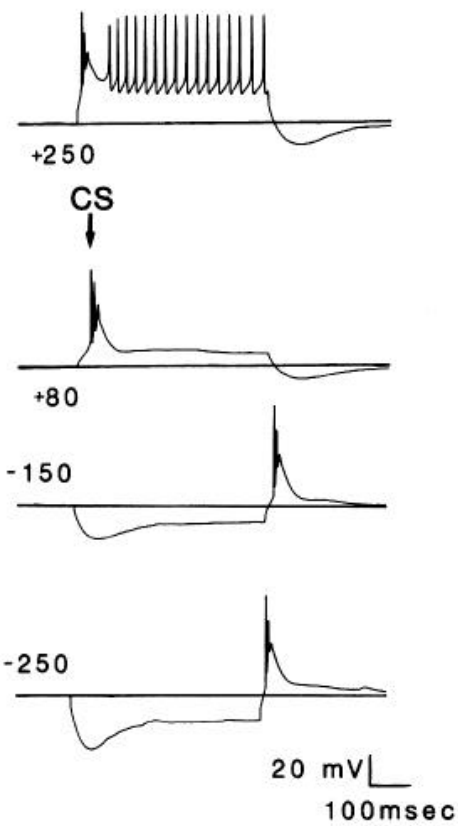

Figure 2. Developmental changes in voltage responses elicited by intracellular injection of depolarizing and hyperpolarizing current pulses. The responses are from four different neurons recorded under current-clamp conditions at the culture age specified. The membrane potentials were held at -62 to $-65 \mathrm{mV}$, considered to reflect the normal resting potential for these neurons. In this and all other current-clamp figures, the number to the left of each voltage trace indicates the amplitude of the applied current pulse, which is not shown. Current pulses were $\sim 500$ msec in duration. In most figures, the spike amplitude is not fully reproduced. Simple spikes $(S S)$ were characteristic of all culture ages. Complex spikes $(C S)$ were a property of older neurons. Doublet spikes $(D S)$ are considered to be an immature form of the complex spike. $O R$, off-response. 
A

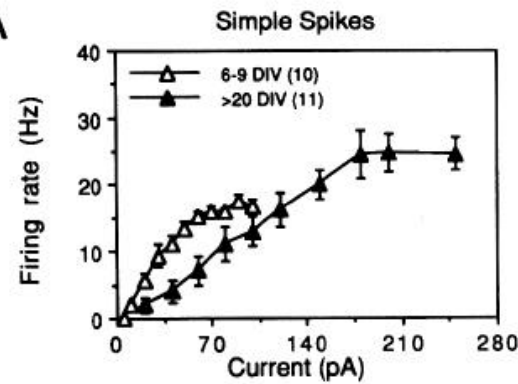

B

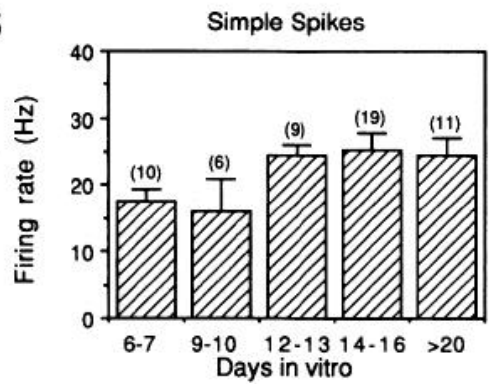

C

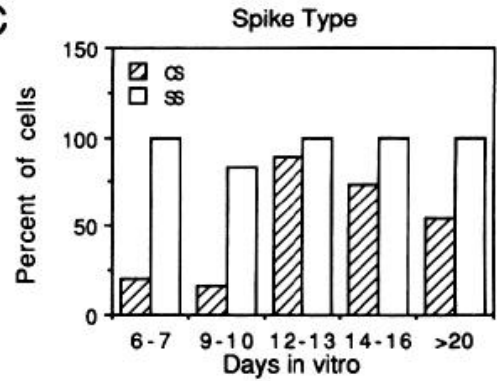

Figure 3. Developmental changes in current evoked spiking. A, The relationship between simple spike firing rate (mean \pm SEM) and current intensity for the population of neurons studied at 6-9 DIV and $>20$ DIV. Spikes were evoked by depolarizing current pulses 500 msec in duration. Spikes in the initial burst discharge (i.e., the complex spike) that preceded the simple spike firing of the older neurons were not included in the count. At all culture ages, firing rate increased to a maximum as the current intensity was increased. Larger currents were necessary for the older neurons because of the lower input resistance (see Fig. 6). Numbers in parentheses indicate the number of neurons studied. B, Maximum firing rate (mean $\pm \mathrm{SEM}$ ) for simple spikes at each culture age studied. The mean firing rate abruptly increased between 9-10 and 12-13 DIV. $C$, The percentage of cells exhibiting simple spikes $(S S)$ and prominent burst events considered to be complex spikes $(C S)$ for the population of neurons studied. The largest increase in neurons exhibiting the complex spike occurred between 9-10 DIV and 12-13 DIV. Data are from the same population of neurons as in $B$.

A1
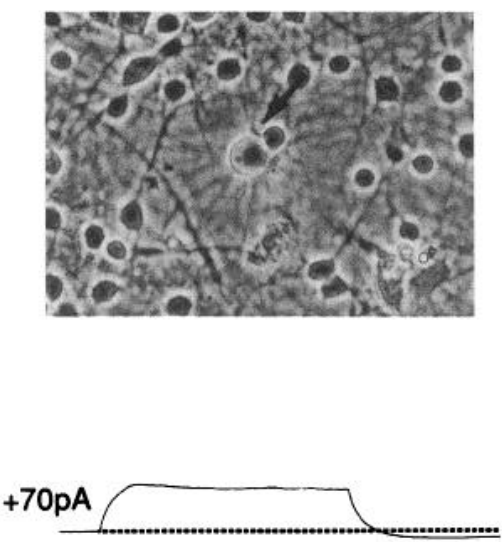

$-70 p A$
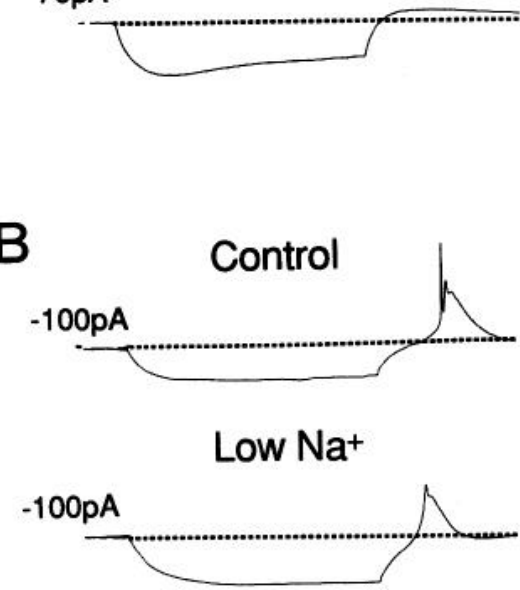

$-150 \mathrm{pA}$

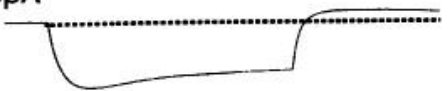

C

A2
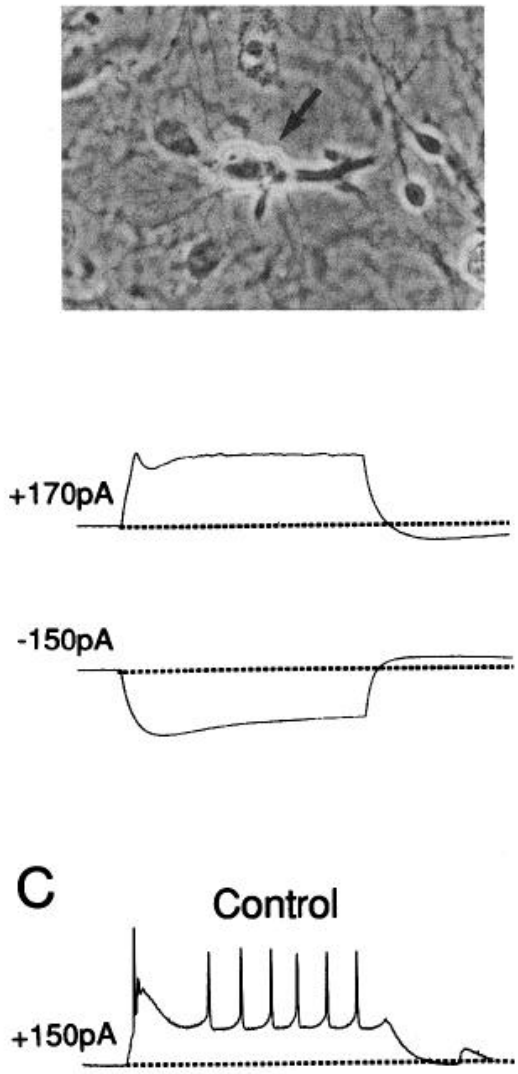

Control

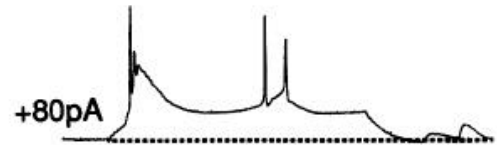

A3

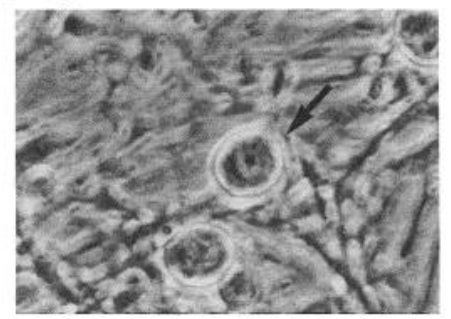

$\overline{20 \mu}$
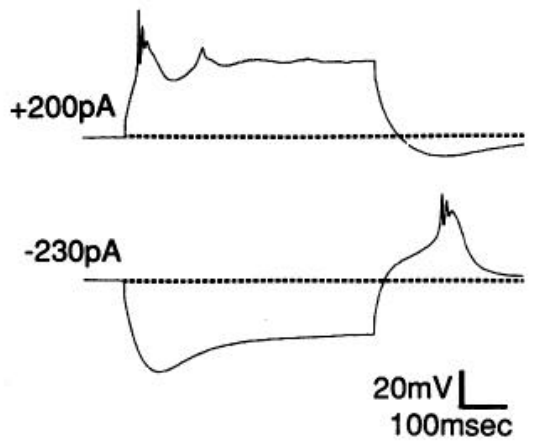

Low $\mathrm{Ca}^{2+}$
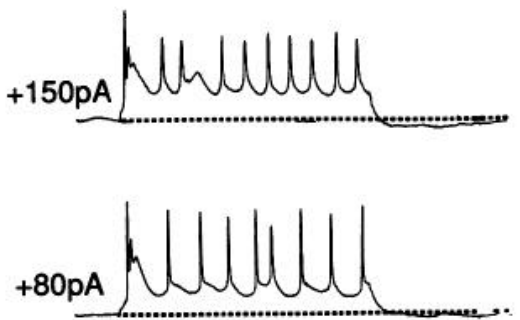

Figure 4. Pharmacological properties of active responses. A, Effect of TTX on current-evoked spike activity. The recordings are from the three neurons shown in the phase-contrast micrographs: $A 1,6 \mathrm{DIV} ; A 2,12 \mathrm{DIV} ; A 3,21 \mathrm{DIV}$. TTX $(1 \mu \mathrm{M})$ was present in the recording medium. TTX blocked the repetitive simple spike firing at all culture ages. The complex spike at the onset of the voltage response in older neurons was not antagonized. Control firing patterns (no TTX) were similar to that shown in Figure 2. B, Replacement of external sodium to $50 \%$ of the control level with isosmotic sucrose blocked the fast spike of the complex spike and reduced the amplitude and duration of the slow depolarization. Total removal of extracellular sodium did not eliminate the slow depolarization (not shown). $C$, Reducing external calcium from $2.2 \mathrm{~mm}$ to approximately $0.2 \mathrm{~mm}$ decreased the amplitude and duration of the complex spike and altered the repetitive simple spike firing pattern. 
A

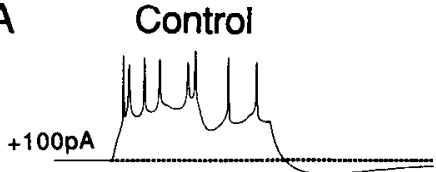

Figure 5. Effect of cadmium on current-evoked spiking. The cadmium was applied by micropressure from a pipette containing $50 \mu \mathrm{M} \mathrm{CdCl}_{2}$ dissolved in bath saline to a 9 DIV Purkinje neuron $(A)$ and a 13 DIV Purkinje neuron $(B)$. Cadmium blocked repetitive firing at both culture ages. The complex spike (arrow) evoked by a hyperpolarizing current pulse in the 13 DIV neuron was not blocked by $50 \mu \mathrm{m}$ cadmium. The smaller-amplitude, long-duration fast spike of the complex spike was blocked by cadmium.

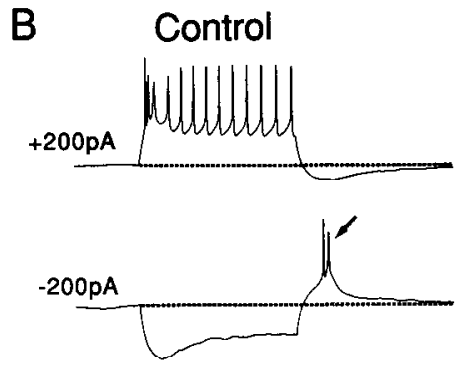

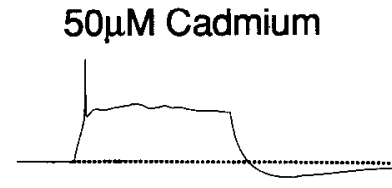
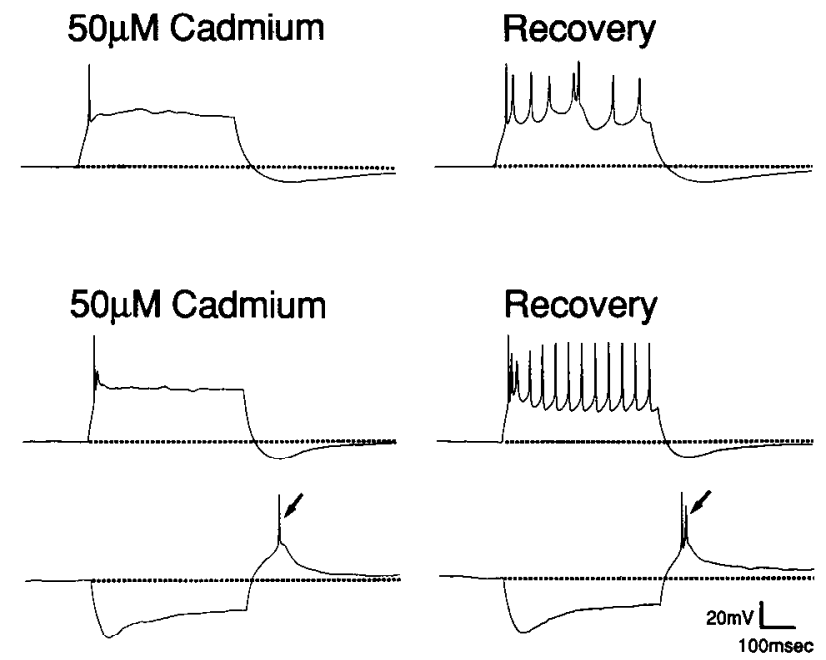

aration and is referred to as such. Based on this similarity, the burst event of the young cultured neurons is considered to be an immature form of the complex spike. The percentage of neurons exhibiting the complex spike increased during the culture period (Fig. 3C). The largest increase occurred between 910 and 12-13 DIV. In the older neurons, small depolarizations evoked this spike event without the subsequent repetitive simple spike activity (Fig. 2), indicating that threshold for activation was at a more hyperpolarized potential than that for repetitive firing. A similar event was evoked at the termination of hyperpolarizing current pulses of sufficient amplitude (Fig. 2) or by depolarization from a hyperpolarized holding potential (see Fig. $6 B)$.

The fast simple spikes in young and old ncurons wcre antagonized by addition of TTX to the recording medium or replacement of extracellular sodium with an impermeant compound (Fig. $4 A, B$ ). The slow depolarization of the complex spike was not blocked but was reduced somewhat in amplitude and duration (Fig. $4 B$ ). Removal of external calcium decreased the amplitude of the complex spike, altered the repetitive simple spike firing, and reduced the amplitude of the simple spikes (Fig. $4 C$ ), an effect not observed with a similar reduction in extracellular magnesium (not shown). Low concentrations of cadmium (50-100 $\mu \mathrm{M})$, a calcium channel blocker, antagonized the repetitive simple spike activity of the young and old neurons but did not block the slow depolarization of the complex spike (Fig. 5). However, $10 \mathrm{~mm}$ magnesium, another calcium channel blocker, antagonized both the complex spike and repetitive simple spike activity (not shown).

\section{Response to hyperpolarizing current pulses}

At all culture ages, the voltage response evoked by hyperpolarizing current pulses exhibited a "sag," a decline in amplitude with time, indicative of the activation of a time-dependent conductance (Fig. 2). A slow membrane depolarization, the offresponse, was produced at the termination of the current pulse. The amplitude of the "sag" and the off-response increased with current intensity (Fig. 6Al,Bl). In young neurons, large offresponses evoked simple spikes; in older neurons, a complex spike was produced (Fig. 2). The amplitude of the off-response, and to a lesser extent the sag, increased during the culture period (Fig. 6A2,B2). The largest increase in the amplitude of the offresponse occurred between 9-10 and 12-13 DIV, reflecting the developmental expression of the complex spike. In contrast, the sag increased gradually between 6-7 and 12-13 DIV.

The sag and the slow depolarization of the off-response were antagonized by $0.5-1 \mathrm{~mm}$ external cesium (Fig. 7), suggesting that a time-dependent inward rectifier (i.e., anomalous rectifier) mediated these responses. Hyperpolarization of the membrane to potentials where anomalous rectifiers are continuously active resulted in a decrease in input resistance (Fig. 8), which was blocked by $0.5-1 \mathrm{~mm}$ external cesium (not shown). At the hyperpolarized holding potential, voltage responses evoked by hyperpolarizing current pulses did not show the sag or off-response evident at resting membrane potential (Fig. 8).

\section{Current-voltage relationships}

The current-voltage relationship changed during the developmental period (Fig. 6Cl). In young cultures (6-7, 9-10 DIV), current-voltage curves were relatively linear over a wide range of membrane potentials. With development, the curves became more S-shaped, reflecting the expression of voltage-sensitive conductances in both the depolarizing and hyperpolarizing range of membrane potentials. Input resistance, measured in the linear range of the hyperpolarizing current-voltage curves near resting membrane potential, decreased during development (Fig. 6C2).

\section{Calcium currents studied under voltage-clamp condition}

The sensitivity of the current-evoked spike activity of the young and old neurons to calcium channel blockers indicated that calcium currents play a prominent role in the generation of these events. Voltage clamp studies were pursued to characterize these currents and to identify developmental changes that could underlie the changes observed in the current-clamp studies. Measurements were made at three culture ages: 7-9 DIV, 12 DIV, and $\geq 14$ DIV. At the older culture ages ( $\geq 12$ DIV) neurons with limited dendritic structure were selected for study, to minimize space-clamp problems. Representative recordings from neurons at early and late developmental stages are shown in Figures 9 and 10. Current-voltage curves, collected at holding potentials of $-40 \mathrm{mV},-62 \mathrm{mV}$, and $-90 \mathrm{mV}$, are shown in Figure 11. Currents evoked at a holding potential of $-62 \mathrm{mV}$ were considered to reflect conductances that could be activated at the normal resting potential of the neuron. Mean current amplitudes at test potentials of $\sim-30 \mathrm{mV}$ and $\sim 0 \mathrm{mV}$ are summarized in Table 1. 
A1

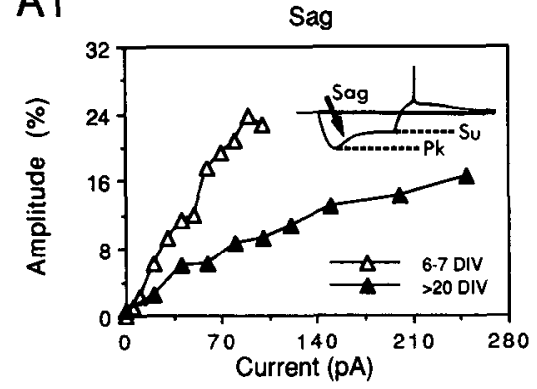

A2

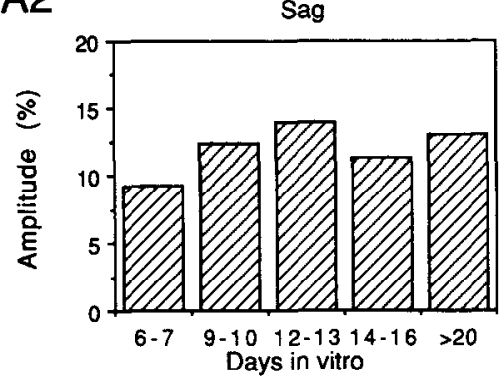

B1

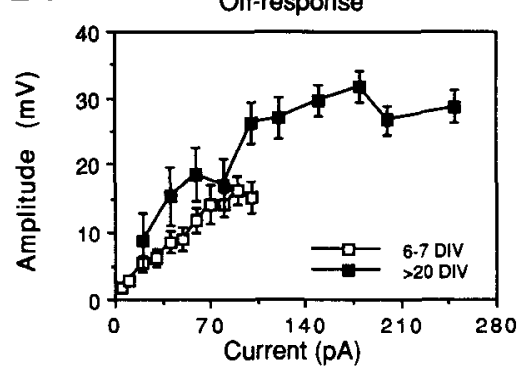

B2

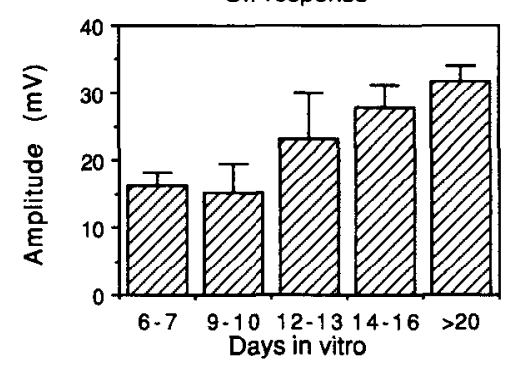

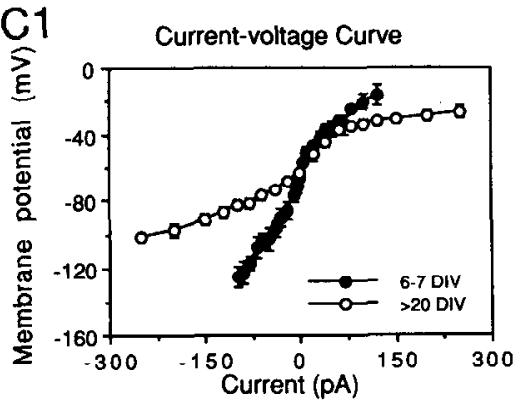

$\mathrm{C} 2$

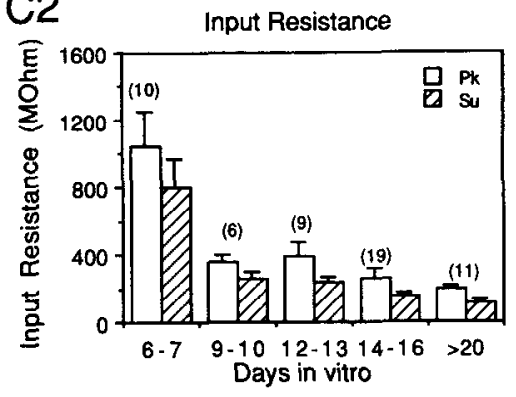

Figure 6. Developmental changes in responses evoked by hyperpolarizing pulses and in the current-voltage relationship. $A 1$, The relationship between the amplitude of the "sag" and current intensity for the population of neurons studied at 6-7 DIV and >20 DIV. The sag was measured as the difference between the mean peak $(P k)$ and sustained $(S u)$ levels of the hyperpolarizing response (see inset) for the population of neurons studied at each culture age and is expressed as a percentage of the peak amplitude. The amplitude of the sag increased with current intensity at all culture ages. $A 2$, The amplitude of the sag (expressed as a percentage of peak amplitude) at different culture ages. Data were obtained from responses that hyperpolarized the membrane to a standard peak value of $-90 \mathrm{mV}$. The amplitude of the sag showed a small increase between 6-7 and 1213 DIV. B1, The relationship between the amplitude (mean \pm SEM) of the off-response and current intensity for the population of neurons studied at 6-7 DIV and $>20$ DIV. The amplitude of the off-response, measured from baseline level to the peak of the depolarization, increased to a maximum as the current intensity was increased. B2, The maximum amplitude of the off-response (mean \pm SEM) at each culture age. Maximum amplitudes were similar at 6-7 and 9-10 DIV and then increased with age, reflecting the developmental expression of the complex spike. $C 1$, Current-voltage relationships constructed from mean $( \pm$ SEM) values for the population of neurons studied at 6-7 DIV and $>20$ DIV. Voltage responses were measured at the initial peak level. Graphs for the sustained level (not shown) were similar. The current-voltage curves were more $\mathrm{S}$-shaped in older neurons. C2, Input resistance (mean $\pm \mathrm{SEM}$ ) for the population of neurons studied at each culture age. Input resistance (slope resistance) was calculated from the linear region of the current-voltage curve near resting potential for both the peak (Pk) and sustained (Su) current-voltage curves. Input resistance decreased with culture age. The largest decrease occurred between 6-7 and 9-10 DIV. Numbers in parenthesis indicate the number of neurons studied, which were the same for all studies illustrated in this figure. Data are from the same population of neurons as shown in Figure 3.

In the young neurons (7-9 DIV) at all three holding potentials, depolarization of the membrane with a series of test commands evoked inward currents characterized by an initial peak that slowly declined to a smaller relatively sustained level, presum- ably reflecting time-dependent inactivation (Fig. 9A). The magnitude of the inactivating component, which was largest for the $-90 \mathrm{mV}$ holding potential, is shown in the difference curves of Figure 11. The inward current activated at potentials around
A

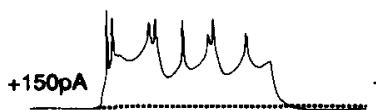

$+50 p \mathrm{~A}$

$-50 \mathrm{pA}$

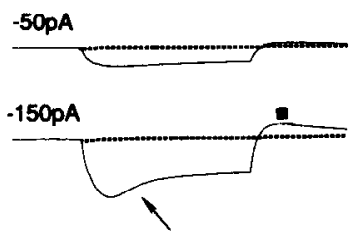

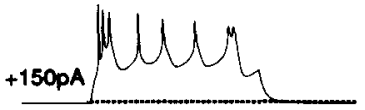

$0.5 \mathrm{mM}$ Cesuim
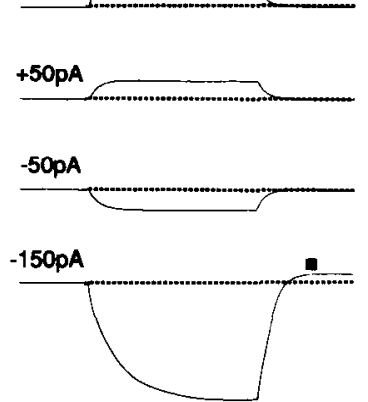

B

Control
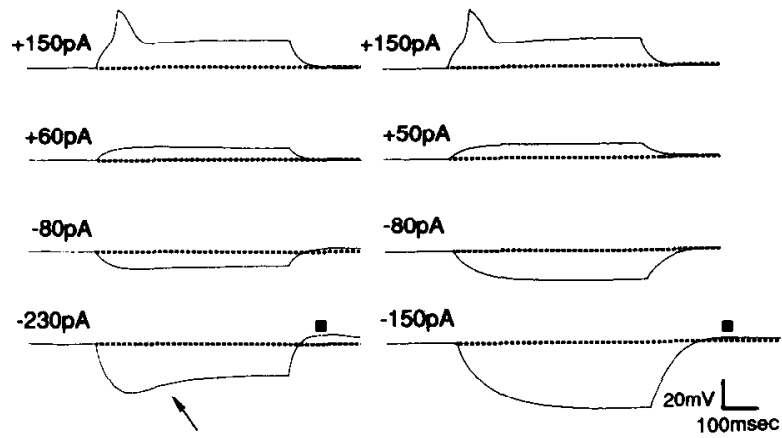

Figure 7. Effect of extracellular cesium on the current-evoked voltage response. Recordings are from a 10 DIV $(A)$ and a 19 DIV $(B)$ Purkinje neuron. In $B$, TTX $(1 \mu \mathrm{M})$ was present in the recording medium. Under control conditions (no TTX), the firing pattern was similar to that shown for the 21 DIV Purkinjc ncuron in Figurc 2. At both culture ages, cesium blocked the sag (arrow) in the voltage response elicited by hyperpolarizing current pulses and increased the amplitude of the response. Cesium also reduced the slow afterdepolarization at the termination of the hyperpolarizing pulse (ם). Voltage responses evoked by depolarizing current pulses were relatively unaffected by cesium. 


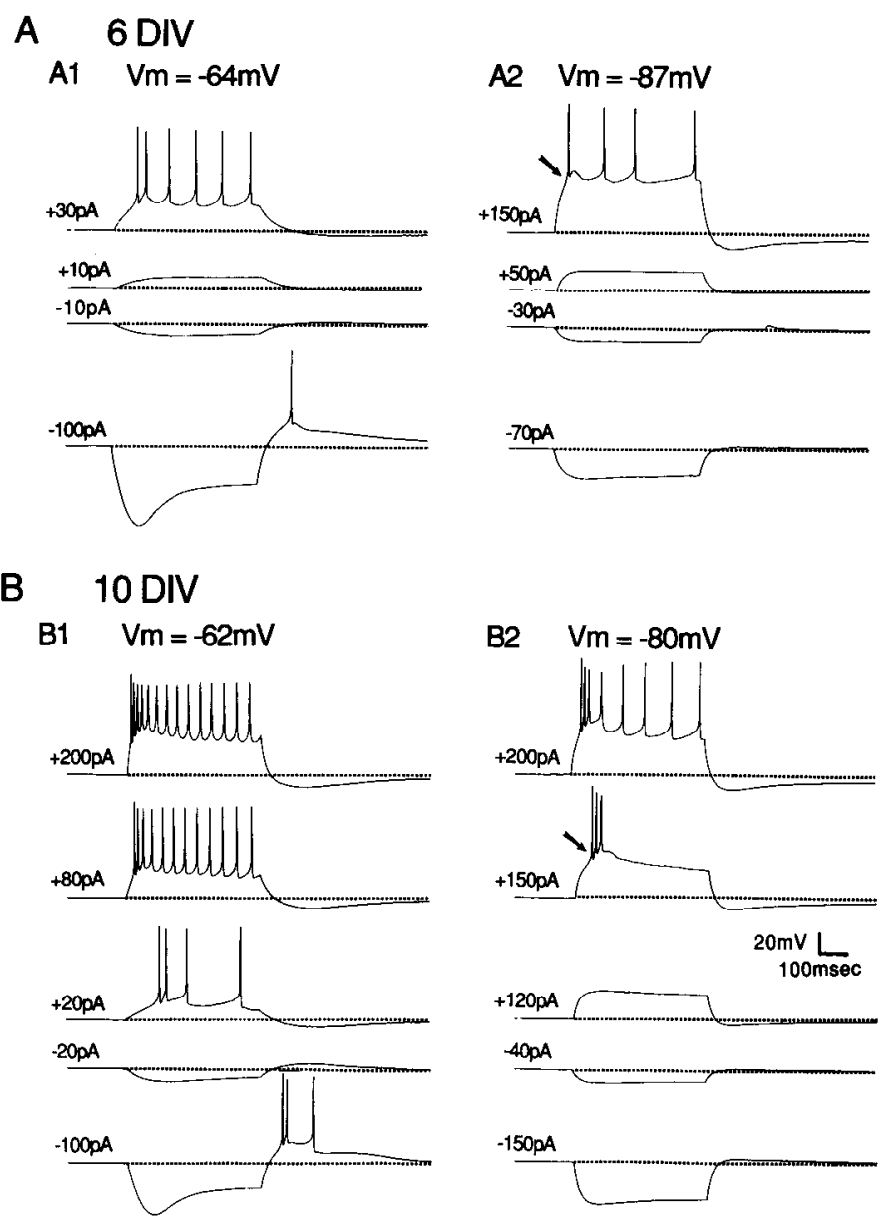

Figure 8. Effect of membrane hyperpolarization on current-voltage relations. Recordings are from a 6 DIV $(A)$ and 10 DIV $(B)$ Purkinje neuron at two membrane potentials, rest $(\sim-62 \mathrm{mV})$ and $\sim 20 \mathrm{mV}$ hyperpolarized from rest $(\sim-80 \mathrm{mV})$. At both culture ages, hyperpolarization of the membrane eliminated the sag in the hyperpolarizing voltage response and the off-response produced at the termination of the current pulse. In the older neuron (10 DIV), depolarization of the membrane from the hyperpolarized potential elicited a burst event ( $\mathrm{ar}$ row) at the onset of the voltage response. A similar burst event (arrow) was not evident in the 6 DIV neuron.
$-20 \mathrm{mV}$ and was largest at potentials around $0 \mathrm{mV}$ (Fig. 11). Low concentrations (50-100 $\mu \mathrm{M})$ of cadmium antagonized the current in a reversible manner (Fig. 9, Table 2).

In older neurons with dendritic structure, an inward calcium current with properties similar to that observed in the young neurons was evident (Fig. 10). Properties in common included a similar voltage sensitivity, time course, and cadmium sensitivity. In addition, a rapidly inactivating current evoked at more hyperpolarized test commands was present (referred to as the low-threshold current; Fig. 10). This current was elicited by depolarizing test commands from holding potentials of $-62 \mathrm{mV}$ or $-90 \mathrm{mV}$ but not from a holding potential of $-40 \mathrm{mV}$. Threshold potential for activation was around $-50 \mathrm{mV}$. At test potentials of $-50 \mathrm{mV}$ to $-40 \mathrm{mV}$, where the current could be observed in isolation, the current reached an initial peak within 20-30 msec of the pulse onset and then declined to approximatcly basclinc level within $100 \mathrm{msec}$. At more depolarized potentials, a sustained component was also evident, presumably reflecting activation of the higher-threshold current described above (referred to as the high-threshold current). In contrast to the high-threshold current, the low-threshold current was resistant to low doses (50-100 $\mu \mathrm{M}$ ) of cadmium (Fig. 10, Table 2). Nickel $(100-300 \mu \mathrm{M})$ and amiloride $(100 \mu \mathrm{M})$ were also relatively ineffective or antagonized both the high- and low-threshold currents (not shown).

\section{Developmental changes in calcium currents}

A comparison of current amplitudes, current-voltage curves, and difference curves revealed developmental changes in the calcium currents (Fig. 11). At all three holding potentials, the amplitude of the inward current both at the initial peak and at the sustained level near the termination of the pulse increased with development. The amplitude of the inactivating component evident in the difference curves (Fig. 11A3-C3) also increased with development, most notably at test potentials where the low-threshold calcium current was observed. In addition, at holding potentials of $-62 \mathrm{mV}$ and $-90 \mathrm{mV}$, the threshold potential for activation of the peak current shifted in the negative direction. This shift did not occur for currents measured at the sustained current level or for peak and sustained currents measured at a holding potential of $-40 \mathrm{mV}$, when the low-threshold

\begin{tabular}{|c|c|c|c|c|c|c|}
\hline \multirow{3}{*}{$\begin{array}{l}\text { Age } \\
\text { (DIV) }\end{array}$} & \multirow[b]{3}{*}{$N$} & \multirow{3}{*}{$\begin{array}{l}\text { Holding } \\
\text { poten- } \\
\text { tial } \\
(\mathrm{mV})\end{array}$} & \multicolumn{4}{|c|}{ Test potential (pA) } \\
\hline & & & \multicolumn{2}{|l|}{$-30 \mathrm{mV}$} & \multicolumn{2}{|l|}{$0 \mathrm{mV}$} \\
\hline & & & $I_{\mathrm{pk}}$ & $I_{\mathrm{su}}$ & $I_{\mathrm{pk}}$ & $I_{\mathrm{su}}$ \\
\hline $7-9$ & 4 & -40 & $-35 \pm 15$ & $-45 \pm 14$ & $-278 \pm 107$ & $-187 \pm 48$ \\
\hline 12 & 2 & -40 & $-24 \pm 10$ & $-34 \pm 10$ & $-393 \pm 37$ & $-354+12$ \\
\hline$\geq 14$ & 10 & -40 & $-70 \pm 14$ & $-95 \pm 19$ & $-637 \pm 127$ & $-541 \pm 106$ \\
\hline $7-9$ & 15 & -62 & $-43 \pm 9$ & $-49 \pm 11$ & $-307 \pm 56$ & $-231 \pm 27$ \\
\hline 12 & 8 & -62 & $-55 \pm 21$ & $-48 \pm 16$ & $-397 \pm 58$ & $-320 \pm 54$ \\
\hline$\geq 14$ & 24 & -62 & $-221 \pm 37^{*}$ & $-71 \pm 13$ & $-543 \pm 68^{*}$ & $-357 \pm 53$ \\
\hline $7-9$ & 9 & -90 & $-64 \pm 31$ & $-61 \pm 24$ & $-647 \pm 146$ & $-387 \pm 68$ \\
\hline 12 & 6 & -90 & $-243 \pm 125^{* *}$ & $-118 \pm 53$ & $-924 \pm 72$ & $-551 \pm 30$ \\
\hline$\geq 14$ & 13 & -90 & $-716 \pm 133^{*}$ & $-368 \pm 107^{*}$ & $-986 \pm 134$ & $-629 \pm 75^{*}$ \\
\hline
\end{tabular}

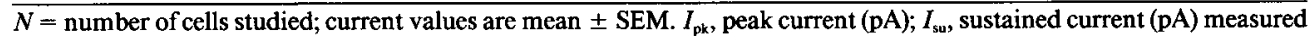
near the termination of the command.

* Statistically significant difference compared to 7-9 DIV value ( $P \leq 0.05$ level, ANOVA).

** Statistically significant compared to $7-9$ and $\geq 14$ DIV values $(P \leq 0.05$ level, ANOVA). 


\section{A Control}

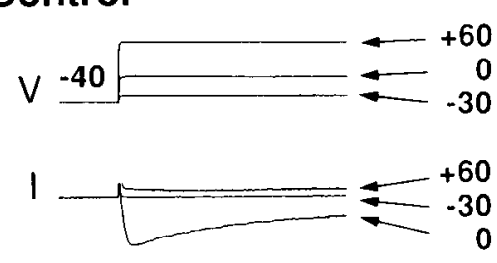

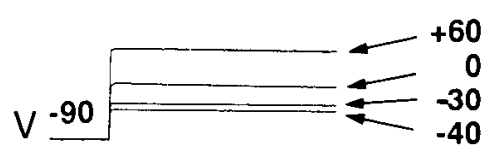

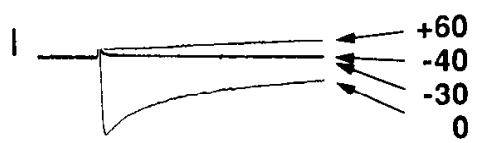

30

60
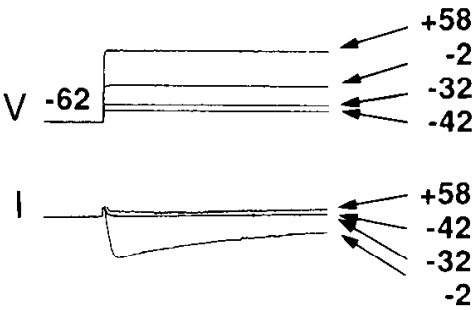

\section{B $50 \mu \mathrm{M}$ Cadmium}

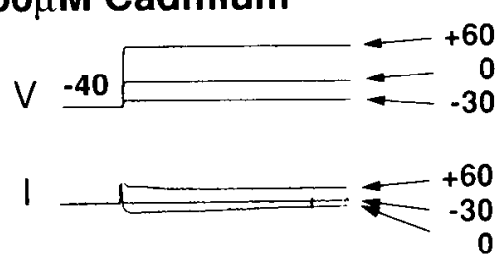

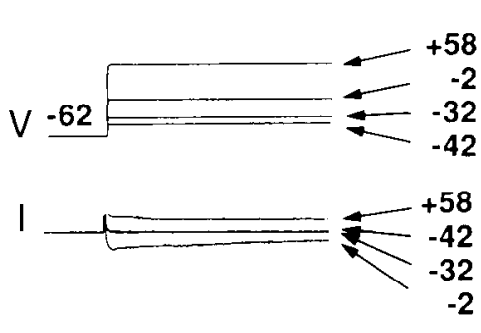

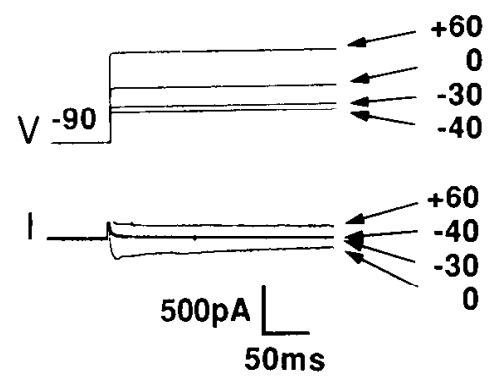

Figure 9. Calcium currents recorded in a 9 DIV Purkinje neuron studied under voltage-clamp conditions. Top traces show the voltage recordings; bottom traces show the current recordings. Numbers to the right indicate the test potentials. $A$, Currents evoked by depolarizing test commands from three different holding potentials. Depolarization from all three holding potentials to test potentials around $-40 \mathrm{mV}$ to $-30 \mathrm{mV}$ evoked only small leak currents. Stronger depolarization, to $0 \mathrm{mV}$, evoked an inward current that reached an initial peak and then declined to a smaller, relatively sustained level. Depolarization to around $+60 \mathrm{mV}$ evoked a small outward current. $B$, Exposure to $50 \mu \mathrm{m}$ cadmium applied by micropressure antagonized the inward current.

current was inactivated. The potential at which the maximum current was evoked also shifted in the negative direction during development, from $\sim 0 \mathrm{mV}$ at $7-9$ DIV to $\sim-15 \mathrm{mV}$ at $>13$ DIV.

\section{Discussion}

Current-clamp recordings from cultured Purkinje neurons at various stages of morphological development demonstrated that the electrophysiological properties changed during maturation. These changes included (1) an increase in firing rate, (2) ex- pression of the complex spike, (3) reshaping of the currentvoltage relationship, and (4) a decrease in input resistance. The decrease in input resistance presumably resulted from the large increase in neuronal size that occurs during development and from the expression of the associated membrane conductances. However, the reshaping of the current-voltage relationship, the increase in firing rate, and the late appearance of the complex spike indicated that the population of ionic conductances also changed during maturation.

The appearance of the complex spike between 9-10 and 12-

Table 2. Effect of cadmium on calcium currents in cerebellar Purkinje neurons

\begin{tabular}{|c|c|c|c|c|c|c|}
\hline \multirow[b]{3}{*}{ Condition } & \multirow[b]{3}{*}{$N$} & \multirow{3}{*}{$\begin{array}{l}\text { Holding } \\
\text { poten- } \\
\text { tial } \\
(\mathrm{mV})\end{array}$} & \multicolumn{4}{|c|}{ Test potential $(\mathrm{pA})$} \\
\hline & & & \multicolumn{2}{|l|}{$-30 \mathrm{mV}$} & \multicolumn{2}{|l|}{$0 \mathrm{mV}$} \\
\hline & & & $\overline{I_{\mathrm{pk}}}$ & $I_{\mathrm{su}}$ & $I_{\mathrm{pk}}$ & $I_{\mathrm{su}}$ \\
\hline \multicolumn{7}{|l|}{7 DIV } \\
\hline Control & 5 & -62 & $-42 \pm 9$ & $-34 \pm 7$ & $-497 \pm 165$ & $-302 \pm 94$ \\
\hline $\begin{array}{l}\text { Cadmium puff } \\
\% \text { Change }\end{array}$ & 5 & -62 & $\begin{array}{l}-41 \pm 8 \\
-2 \%\end{array}$ & $\begin{array}{c}-35 \pm 8 \\
+3 \%\end{array}$ & $\begin{array}{l}-109 \pm 51 \\
-78 \%\end{array}$ & $\begin{array}{c}-108 \pm 3 \\
-64 \%\end{array}$ \\
\hline Control & 3 & -90 & $-17 \pm 26$ & $-33 \pm 8$ & $-776 \pm 264$ & $-361 \pm 109$ \\
\hline $\begin{array}{l}\text { Cadmium puff } \\
\% \text { Change }\end{array}$ & 3 & -90 & $\begin{array}{l}-49 \pm 12 \\
+188 \%\end{array}$ & $\begin{array}{l}-29 \pm 8 \\
-12 \%\end{array}$ & $\begin{array}{l}-244 \pm 89 \\
-69 \%\end{array}$ & $\begin{array}{l}-186 \pm 44 \\
-48 \%\end{array}$ \\
\hline \multicolumn{7}{|l|}{$\geq 14 \mathrm{DIV}$} \\
\hline Control & 10 & -62 & $-258 \pm 39$ & $-55 \pm 7$ & $-818 \pm 130$ & $-551 \pm 113$ \\
\hline $\begin{array}{l}\text { Cadmium puff } \\
\% \text { Change }\end{array}$ & 10 & -62 & $\begin{array}{c}-173+25 \\
-33 \%\end{array}$ & $\begin{array}{l}-42 \pm 9 \\
-24 \%\end{array}$ & $\begin{array}{c}-293 \pm 85 \\
-64 \%\end{array}$ & $\begin{array}{l}-160 \pm 57 \\
-70 \%\end{array}$ \\
\hline Control & 3 & -90 & $-221 \pm 94$ & $-125 \pm 36$ & $-822 \pm 195$ & $-514 \pm 195$ \\
\hline $\begin{array}{l}\text { Cadmium puff } \\
\% \text { Change }\end{array}$ & 3 & -90 & $\begin{array}{c}-209 \pm 96 \\
-5 \%\end{array}$ & $\begin{array}{l}-128 \pm 55 \\
+2 \%\end{array}$ & $\begin{array}{l}-254 \pm 81 \\
-69 \%\end{array}$ & $\begin{array}{l}-130 \pm 12 \\
-75 \%\end{array}$ \\
\hline
\end{tabular}

$N=$ number of cells studied; current values are mean \pm SEM. Percentage change was calculated from mean values.

$I_{\mathrm{pk}}$, peak current $(\mathrm{pA}) ; I_{\mathrm{su}}$, sustained current $(\mathrm{pA})$ measured near the termination of the command. 
A
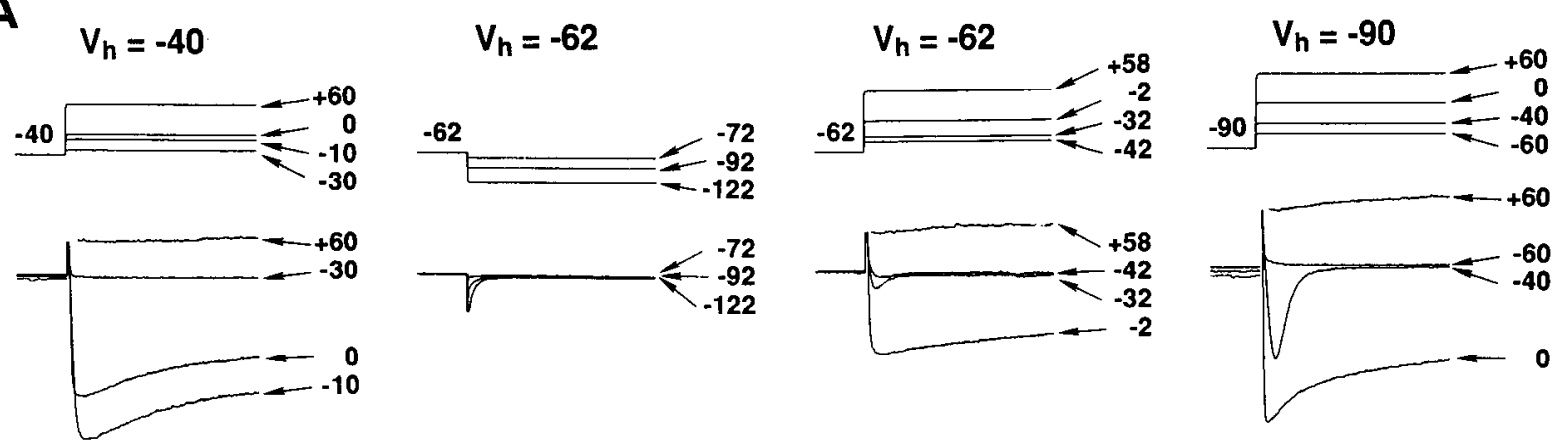

$\mathbf{8}$

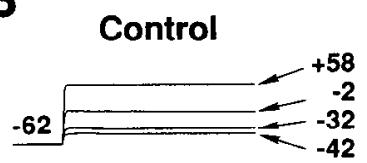

\section{$50 \mu \mathrm{M}$ Cadmium}
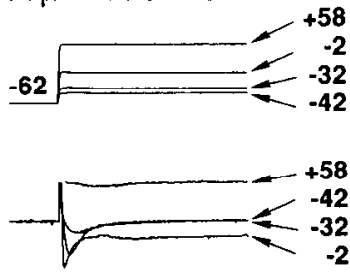

C

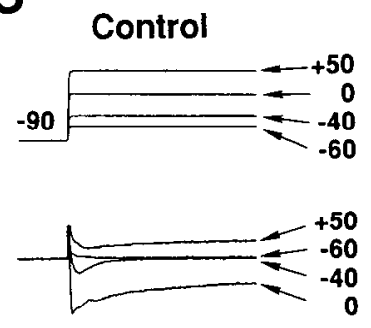

50 4 M Cadmium
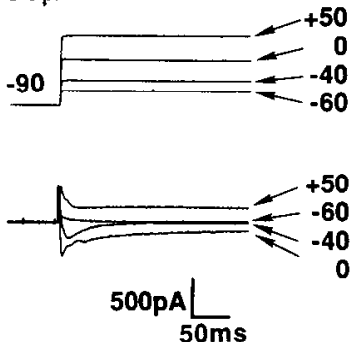

Figure 10. Calcium currents recorded in a 17 DIV Purkinje neuron studied under voltage-clamp conditions. Top traces show the voltage recordings; bottom traces show the current recordings. Numbers to the right indicate the test potentials. $A$, Currents evoked by test commands from three different holding potentials. Depolarization from a holding potential of $-40 \mathrm{mV}$ to a test potential of $-30 \mathrm{mV}$ produced only a small leak current. Depolarization to test potentials of -10 and $0 \mathrm{mV}$ evoked the high-threshold current. Depolarization from a holding potential of $-62 \mathrm{mV}$ or -90 $\mathrm{mV}$ evoked both a low-threshold rapidly inactivating current and the high-threshold sustained current. The low-threshold current is evident in the traces for test potentials to around $-40 \mathrm{mV}$. The high-threshold current is evident in the traces for test potentials to around $0 \mathrm{mV}$. An outward current is evoked by the most depolarized test potentials. Hyperpolarizing commands from a holding potential of $-62 \mathrm{mV}$ elicited a very small lcak current following the capacitance tansient. $B$ and $C$, Voltage-clamp recordings from two 14 DIV Purkinje neurons studied at different holding potentials, $-62 \mathrm{mV}(B)$ and $-90 \mathrm{mV}(C)$. Inward currents similar to that shown for the cell in $A$ were observed. Exposure to $50 \mu \mathrm{M}$ cadmium applied by micropressure antagonized the high-threshold but not the low-threshold current.

13 DIV was one of the most prominent developmental changes observed in the current-clamp studies. Studies with calcium channel blockers and ion substitution indicated that calcium conductances play a major role in the generation of this spike event, although sodium and potassium conductances may also contribute. In voltage-clamp studies of older neurons that expressed the complex spike, two calcium currents were identified: (1) a high-threshold slowly inactivating calcium current sensitive to 50-100 $\mu \mathrm{M}$ external cadmium, and (2) a low-threshold rapidly inactivating calcium current relatively resistant to these concentrations of cadmium. In the young Purkinje neurons, which did not express the complex spike, only the high-threshold current was evident. The properties of these currents were similar to those described previously for high- and low-threshold currents in cultured (Bossu et al., 1990) and acutely isolated Purkinje neurons (Regan et al., 1991; Regan, 1991), and in other CNS neurons (Kay and Wong, 1987; Yaari et al., 1987; Lux, 1988; Tsien et al., 1988; Coulter et al., 1989; Crunelli et al., 1989; McCobb et al., 1989, 1990; Meyers and Barker, 1989; Suzuki and Rogawski, 1989; Takahashi et al., 1989; Hamill et al., 1991). However, the low-threshold current in the cultured Purkinje neurons was relatively resistant to low doses of nickel and amiloride, compounds reported to block the low-threshold calcium current in some preparations (Narahashi et al., 1987; Tang et al., 1988; Tsien et al., 1988; McCobb et al., 1990).

The time course, voltage sensitivity, and pharmacology of the low-threshold current were similar to that observed for the slow depolarization of the complex spike. The developmental ex- pression of the low-threshold current and the complex spike occurred over a similar time period. Thus, the low-threshold calcium current appears to play a prominent role in the generation of this spike event. A contribution of the high-threshold calcium current also seems likely, based on studies of Purkinje neurons in the slice preparation (Llinas and Sugimori, 1980a,b), but this could not be assessed under the conditions used in the present study.

The developmental expression of the low-threshold calcium current paralleled dendritic development, as did the increase in amplitude of the high-threshold current, which was present before dendrites formed. This correlation suggests that both conductances are present in the dendritic region. A somatic location is also likely, since the high-threshold current was observed in the young neurons without dendrites. Others have also reported that calcium conductances are present in both the somatic and dendritic regions of the Purkinje neurons. Bossu et al. (1989, 1990), using voltage-clamp and single-channel recordings, observed both the low- and high-threshold calcium currents in the somatic and dendritic regions of Purkinje neurons maintained under different culture conditions. Regan (1991) showed that acutely isolated Purkinje neurons from $1-3$ wcck postnatal rats exhibited a high- and a low-threshold calcium current when studied under voltage-clamp conditions. These neurons consisted primarily of the somatic region, although some dendritic structure was present. A dendritic location of the high-threshold calcium current was shown in intracellular recordings from adult Purkinje neurons in slice preparations (Llinas and Sugimori, 
A1

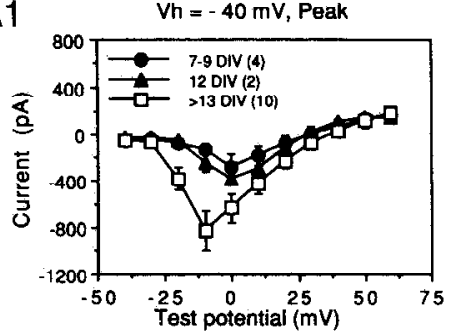

A2

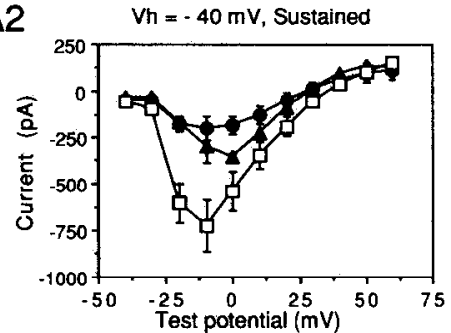

A3

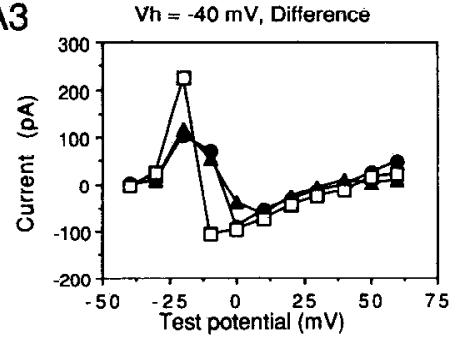

B1

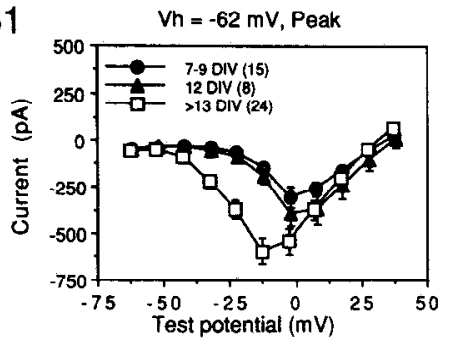

B2

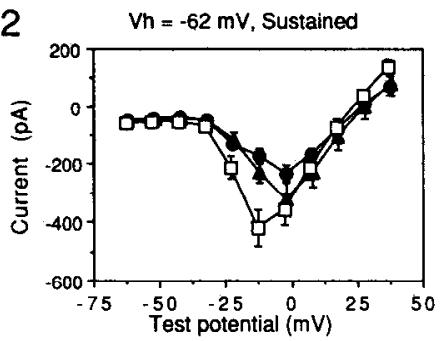

B3

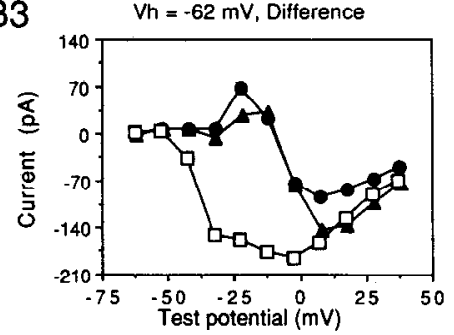

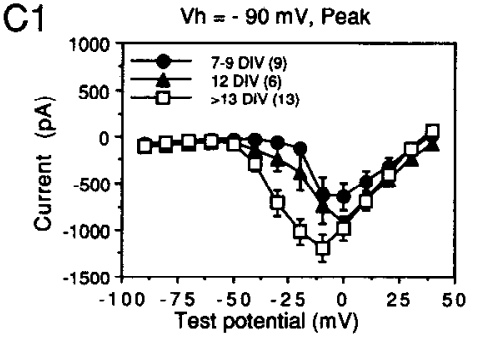

$\mathrm{C} 2$
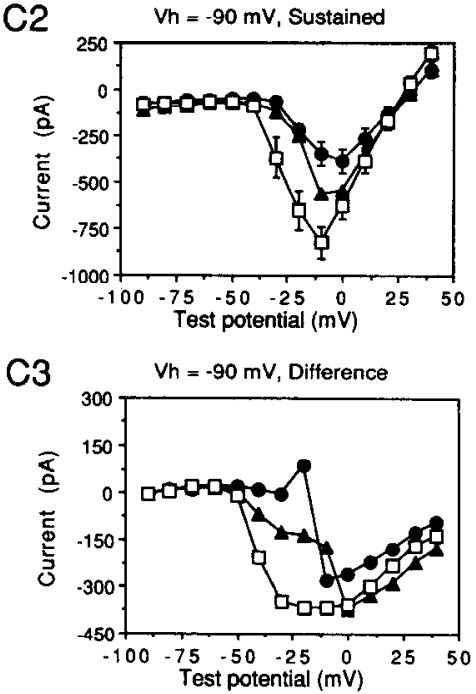

Figure 11. Current-voltage curves for neurons studied under voltage-clamp conditions. Currents were evoked by depolarizing test commands from holding potentials of $-40 \mathrm{mV}(A),-62 \mathrm{mV}(B)$, and $-90 \mathrm{mV}(C)$. Measurements were made of the initial peak amplitude and the amplitude near the termination of the pulse where the current level was relatively stable (referred to as the sustained level). $A I-C 1$, Current-voltage curves constructed from peak values (mean $\pm \mathrm{SEM}$ ). $A 2-C 2$, Current-voltage curves constructed from the sustained values (mean \pm SEM). $A 3-C 3$, Difference curves obtained by subtraction of the peak current amplitude minus the sustained current amplitude at each test potential. Three culture

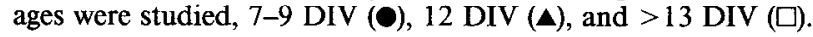

1980 b). In another study, the low-threshold calcium current was identified in Purkinje neurons in the slice preparation (Crepel and Soria-P, 1986), although the cellular localization was not assessed.

Dendritic calcium conductances are thought to play a prominent role in repetitive firing and the generation of spontaneous activity of mature Purkinje neurons (Llinas and Sugimori, 1980b). Results from the current studies support this role. However, in the cultured Purkinje neurons, repetitive firing was evident at youngest age studied, when spontaneous firing patterns were also seen (Gruol and Franklin, 1987). These young neurons did not possess dendrites, and thus dendrites are not absolutely required for this activity. The repetitive firing in the young neurons was antagonized by low concentrations of cadmium (50-100 $\mu \mathrm{M})$, consistent with a role for the high-threshold calcium conductance that was present at that age. The firing rate increased abruptly between 9-10 DIV and 12-13 DIV, coincident with dendritic development, as did the amplitude of the high- and low-threshold calcium currents. These results are consistent with an influence of dendritic calcium conductances on firing rate of the Purkinje neurons.

The complex spike was a prominent component of the current-evoked voltage response in the older cultured neurons, and resembled complex spikes evoked by synaptic input to Purkinje neurons studied in the slice preparation (Llinas and Sugimori, 1980a, 1982; Crepel et al., 1981; Hounsgaard and Midtgaard, 1989). However, Purkinje neurons in the slice preparation do not normally develop a complex spike when depolarized by current pulses. Burst discharges are produced by depolarizing current, but appear later in the response and do not resemble the synaptically evoked complex spike. This dissimilarity between the cultured and slice neurons could result from technical or physiological factors. Whole-cell patch recordings were used for the culturcd ncurons; conventional intracellular methods were used in the slice studies. Ionic conditions and recording temperatures also differed. Additionally, the size and morphological structure of the dendritic trees were not equivalent. The dendritic structure of the cultured neurons is smaller and less complex than that found in mature Purkinje neurons differentiated in vivo. Thus, the applied current may be more effective in depolarizing the former's dendrites, yielding a larger contribution of dendritic conductances to the voltage response. The limited dendritic structure of the cultured neurons could also contribute to their somewhat lower firing rates compared to Purkinje neurons recorded in vivo.

The developmental sequence in expression of the calcium currents in the Purkinje neurons, with an early appearance of the high-threshold calcium current followed later by the lowthreshold current, resembles the developmental sequence reported for neocortical ncurons (Hamill et al., 1991). IIowever, in both hippocampal (Yaari et al., 1987; but see Meyers and Barker, 1989) and spinal cord neurons (McCobb et al., 1989, 1990) this sequence is reversed, with the low-threshold current preceding the high-threshold calcium current. Thus, the role of these two calcium currents in developing CNS neurons may vary according to neuronal type. 
The young Purkinje neurons also exhibited a prominent potassium conductance, identified as the anomalous rectifier based on voltage sensitivity and pharmacological sensitivity. This conductance produced a "sag" in the voltage response to hyperpolarizing current pulses. Similar responses in Purkinje neurons in a slice preparation were shown by voltage clamp to be mediated by a time-dependent anomalous rectifier (Crepel and Soria-P, 1986). The presence of this conductance in the young neurons indicates an early timetable for developmental expression. Kapoor et al. (1988) have also noted the early expression of this conductance in Purkinje neurons studied a slice-culture preparation. The amplitude of the "sag" increased slightly during the culture period, but did not show the dramatic developmental changes observed for the calcium conductances.

\section{References}

Altman J (1972) Postnatal development of the cerebellar cortex in the rat. J Comp Neurol 145:399-464.

Bossu JL, Dupont J-L, Feltz A (1989) Calcium currents in rat cerebellar Purkinje cells maintained in culture. Neuroscience 30:605-617.

Bossu JL, Fagni L, Feltz A (1990) Voltage-activated calcium channels in rat Purkinje cells maintained in culture. Pfluegers Arch 414:1-3.

Calvet M, Calvet J, Eude D, Dufosse M (1985) Morphologic and functional abnormalities that develop in kitten Purkinje neurons during maintenance for months after maturation in organotypic cultures. Brain Res 341:205-221.

Chan C, Hounsgaard J, Midtgaard J (1989) Excitatory synaptic responses in turtle cerebellar Purkinje cells. J Physiol (Lond) 409:143156.

Coulter D, Huguenard J, Prince D (1989) Calcium currents in rat thalamocortical relay neurones: kinetic properties of the transient, low threshold current. J Physiol (Lond) 414:587-604.

Crepel F (1972) Maturation of the cerebellar Purkinje cells. I. Postnatal evolution of the Purkinje cell spontaneous firing in the rat. Exp Brain Res 14:463-472.

Crepel F, Soria-P J (1986) Inward rectification and low threshold calcium conductance in rat cerebellar Purkinje cells. An in vitro study. J Physiol (Lond) 372:1-23.

Crepel F, Dhanjal S, Garthwaite J (1981) Morphological and electrophysiological characteristics of rat cerebellar slices maintained in vitro. J Physiol (Lond) 316:127-138.

Crunelli V, Lightowler S, Pollard C (1989) A T-type Ca ${ }^{2+}$ current underlies low threshold $\mathrm{Ca}^{2+}$ potentials in cells of the cat and rat lateral geniculate nucleus. J Physiol (Lond) 413:543-561.

Gahwiler BH, Llano I (1989) Sodium and potassium conductances in somatic membranes of rat Purkinje cells from organotypic cerebellar cultures. J Physiol (Lond) 417:105-122.

Gruol DL (1983) Cultured cerebellar neurons: endogenous and exogenous components of Purkinje cell activity and membrane response to putative transmitters. Brain Res 263:223-241.

Gruol DL, Crimi CP (1988) Morphological and physiological properties of rat cerebellar neurons in mature and developing cultures. Dev Brain Res 41:135-146.

Gruol DL, Franklin CL (1987) Morphological and physiological differentiation of Purkinje neurons in cultures of rat cerebellum. J Neurosci 7:1271-1293.

Gruol DL, Jacquin T, Yool AJ (1991) Single channel $\mathrm{K}^{+}$currents recorded from the somatic and dendritic regions of cerebellar Purkinje neurons in culture. $\mathrm{J}$ Neurosci 11:1002-1015.

Hamill OP, Huguenard JR, Prince DA (1991) Patch-clamp studies of voltage-gated currents in identified neurons of the rat cerebral cortex. Cerebr Cortex 1:48-61.

Hirano T, Ohmori H (1986) Voltage gated and synaptic currents in rat Purkinje cells in dissociated cell cultures. Proc Natl Acad Sci USA 83:1945-1949.

Hockberger PE, Tseng HY, Connor JA (1989a) Development of rat cerebellar Purkinje cells: electrophysiological properties following acute isolation and in long-term culture. J. Neurosci 9:2258-2271.

Hockberger PE, Tseng HY, Connor JA (1989b) Fura-2 measurements of cultured rat Purkinje neurons show dendritic localization of $\mathrm{Ca}^{2+}$ influx. J Neurosci 9:2272-2284.

Hounsgaard J, Midtgaard J (1989) Synaptic control of excitability in turtle cerebellar Purkinje cells. J Physiol (I ond) 409:157-170.
Hounsgaard J, Midtgaard J (1990) Intrinsic determinants of firing pattern in Purkinje cells of the turtle cerebellum in vitro. J Physiol (Lond) 402:731-749.

Kapoor R, Jaeger CB, Llinas R (1988) Electrophysiology of the mammalian cerebellar cortex in organ culture. Neuroscience 26:493-507.

Kay AR, Wong RKS (1987) Calcium current activation kinetics in isolated pyramidal neurones of the CAl region of the mature guinea pig hippocampus. J Physiol (Lond) 392:603-616.

Llinas R, Sugimori M (1980a) Electrophysiological properties of in vitro Purkinje cells somata in mammalian cerebellar slices. J Physiol (Lond) 305:171-195.

Llinas R, Sugimori M (1980b) Electrophysiological properties of in vitro Purkinje cell dendrites in mammalian cerebellar slices. J Physiol (Lond) 305:197-213.

Llinas R, Sugimori M (1982) Functional significance of the climbing fibre input to Purkinje cells: an in vitro study in mammalian cerebellar slices. Exp Brain Res 61:189-194.

Lux H (1988) Studies on the development of voltage activated calcium channels in vertebrate neurons. In: Calcium and ion channels modulation (Grinnell AD, Armstrong D, Jackson MB, eds), pp 313-324. New York: Plenum.

Marshall K, Wojtowicz J, Hendelman W (1980) Patterns of functional synaptic connections in organized cultures of cerebellum. Neuroscience 5:1847-1857.

McCobb D, Best P, Beam K (1989) Development alters the expression of calcium currents in chick limb motoneurons. Neuron 2:1633-1643.

McCobb DP, Best PM, Beam KG (1990) The differentiation of excitability in embryonic chick limb motoneurons. J Neurosci 10:2974 2984.

Meyers DR, Barker J (1989) Whole cell patch clamp analysis of voltage dependent calcium conductances in cultured embryonic rat hippocampal neurons. J Neurophysiol 61:467-477.

Moonen G, Neale E, MacDonald R, Gibbs W, Nelson P (1982) Cerebellar macroneurons in microexplant cell culture. Methodology, basic electrophysiology, and morphology after horseradish peroxidase injection. Dev Brain Res 5:59-73.

Narahashi T, Tsunoo A, Yoshii M (1987) Characterization of two types of calcium channels in mouse neuroblastoma cells. J Physiol (Lond) 383:231-249.

Regan L (1991) Voltage-dependent calcium currents in Purkinje cells from rat cerebellar vermis. J Neurosci 11:2259-2269.

Regan L, Sah D, Bean B (1991) $\mathrm{Ca}^{2+}$ channels in rat central and peripheral neurons: high threshold current resistant to dihydropyridine blockers and $\omega$-conotoxin. Neuron 6:269-280.

Ross W, Werman R (1987) Mapping calcium transients in the dendrites of Purkinje cells from the guinea pig cerebellum in vitro. $\mathrm{J}$ Physiol (Lond) 389:319-336.

Seil F (1979) Cerebellum in tissue culture. Rev Neurosci 4:105-177.

Suzuki S, Rogawski M (1989) T type calcium channels mediate the transition between tonic and phasic firing in thalamic neurons. Proc Natl Acad Sci USA 86:7228-7232.

Takahashi K, Wakamori M, Akaike N (1989) Hippocampal CA1 pyramidal cells of rats have four voltage-dependent calcium conductances. Neurosci Lett 104:229-234.

Tang C, Presser F, Morad M (1988) Amiloride selectively blocks the low threshold (T) calcium channel. Science 240:213-215.

Tank DW, Sugimori M, Connor JA, Llinas RR (1988) Spatially resolved calcium dynamics of mammalian Purkinje cells in cerebellar slice. Science 242:773-777.

Tsien R, Lipscombe D, Madison D, Bley K, Fox A (1988) Multiple types of neuronal calcium channels and their selective modulation. Trends Neurosci 11:431-438.

Weber A, Schachner M (1984) Maintenance of immunocytologically identified Purkinje cells from mouse cerebellum in monolayer culture. Brain Res 311:119-130.

Woodward DJ, Hoffer BJ, Lapham LW (1969a) Correlative survey of electrophysiological, neuropharmacological, and histochemical aspects of cerebellar Imaturation in rat. In: Neurobiology of cerebellar evolution and development (Llinas R, ed), pp 725-741. Chicago: Amer Med Assn Educ and Res Fdn.

Woodward DJ, Hoffer BJ, Lapham LW (1969b) Postnatal development of electrical and enzyme histochemical activity in Purkinje cells. Exp Neurol 23:120-139.

Yaari Y, Hamon B, Lux HD (1987) Development of two types of calcium channels in cultured mammalian hippocampal neurons. Science 235:680-682. 